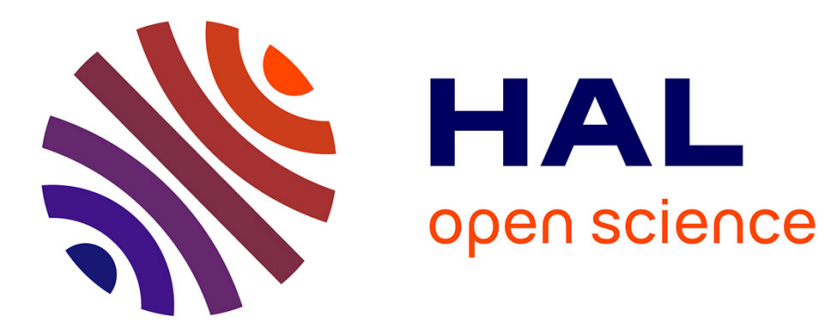

\title{
Comparaison des besoins en éléments minéraux de différentes espèces de graminées à gazon
}

\author{
Francis Lemaire, Jean-Louis Papin
}

\section{To cite this version:}

Francis Lemaire, Jean-Louis Papin. Comparaison des besoins en éléments minéraux de différentes espèces de graminées à gazon. Agronomie, 1985, 5 (1), pp.39-46. hal-00884731

\section{HAL Id: hal-00884731 \\ https://hal.science/hal-00884731}

Submitted on 1 Jan 1985

HAL is a multi-disciplinary open access archive for the deposit and dissemination of scientific research documents, whether they are published or not. The documents may come from teaching and research institutions in France or abroad, or from public or private research centers.
L'archive ouverte pluridisciplinaire HAL, est destinée au dépôt et à la diffusion de documents scientifiques de niveau recherche, publiés ou non, émanant des établissements d'enseignement et de recherche français ou étrangers, des laboratoires publics ou privés. 


\title{
Comparaison des besoins en éléments minéraux de différentes espèces de graminées à gazon
}

\author{
Francis LEMAIRE \\ avec la collaboration technique de Jean-Louis PAPIN \\ I.N.R.A., Station d'Agronomie, Beaucouzé, F 49000 Angers
}

\begin{abstract}
Afin de mieux définir la fertilisation minérale des gazons, les exigences en éléments majeurs de 6 espèces de graminées à gazon : Lolium perenne L. cv. « Manhattan », Festuca rubra L. cv. « Manoir », Poa pratensis L. cv. " Monopoly », Phleum bertelonii L. cv. « Sport ", Festuca arundinacea Schreb. cv. " Ludion ». Agrotis tenuis Sibth., ont été déterminées au moyen de courbes de réponse à l'azote, au phosphore et au potassium. Ces courbes de réponse sont obtenues à l'aide d'une technique de culture en solutions nutritives sur substrat inerte avec aération périodique automatique.

L'utilisation de modèles de régression non linéaire permet de calculer la dose responsable de l'optimum de production, ce qui constitue la base de la comparaison entre les 6 espèces étudiées.

Le classement obtenu permet de proposer des groupes d'espèces à exigences semblables en azote, phosphore et
\end{abstract} potassium, ce qui devrait avoir des conséquences pour la définition des mélanges selon les utilisations.

Mots clés additionnels : $N, P, K$, Lolium perenne, Festuca rubra, Poa pratensis, Phleum bertelonii, Festuca arundinacea, Agrostis tenuis.

In order to estimate better the mineral fertilization of turf, the mineral requirements of 6 turfgrass species : Lolium perenne L. cv. "Manhattan », Festuca rubra L. cv. " Manoir ", Poa pratensis L. cv, " Monopoly ", Phleum bertelonii L. cv. "Sport ", Festuca arundinacea Schreb. cv. "Ludion ", Agrostis tenuis Sibth. were determined with response curves to nitrogen, phosphorus and potassium. The curves were obtained with a growing technique based on liquid feeding on an inert substrate, with automated periodic aeration. By use of non-linear regression models, it was possible to calculate the concentrations responsible for optimum yield, which was the basis of the comparison between the 6 studied species. The classification obtained led us to propose groups of species with similar requirements for nitrogen, phosphorus or potassium.

Additional key words : $N, P, K$, Lolium perenne, Festuca rubra, Poa pratensis, Phleum bertelonii, Festuca arundinacea, Agrostis tenuis.

\section{INTRODUCTION}

Les gazons occupent des surfaces importantes en France : 374605 ha d'après CAIROL et al. (1982). Quels que soient leurs buts (ornemental, sportif, espaces verts publics, fixation), les gazons sont des peuplements plurispécifiques de graminées maintenues à l'état végétatif par des tontes fréquentes. Ces particularités rendent difficile l'extrapolation des résultats obtenus pour les exportations minérales des plantes fourragères au cas des gazons subissant des tontes intensives à moins de considérer les gazons comme un cas limite de prairie surpâturée plus ou moins fertilisée suivant les cas.
Les exigences minérales des différentes espèces de graminées à gazon sont connues à des degrés divers : les besoins en azote (dans l'ordre décroissant, raygrass anglais, agrostis, fétuque élevée, pâturin des prés, fétuque rouge) sont en général mieux déterminés que ceux des autres éléments (phosphore et potassium) (BEARD, 1973) ; certaines espèces comme ray-grass anglais, pâturin des prés, agrostis, ont été plus étudiées que d'autres comme fétuque rouge, fléole (MADISON, 1962 ; Goss \& LAW, 1967 ; LEE, 1976 ; ADAMS, 1977 ; CHRISTIANS et al., 1981 ; DUNN et al., 1981). Pour certaines espèces, les études ont été poussées jusqu'au cultivar. D'après SCHWEIZER (1974), agrostis, fléole, ray-grass anglais et pâturin des prés sont 
plus exigeants en azote et potassium que la fétuque rouge, alors que MINDERHOUD (1976) trouve que la fétuque rouge et l'agrostis ont des besoins moindres en phosphore et potassium.

La plupart des précédents résultats ont été obtenus à l'aide d'essais au champ. L'emploi d'essais en solutions nutritives est réservé à la mise en évidence de l'effet de certaines variables de l'alimentation minérale : NITTLER \& KENNY (1976) ont étudié l'incidence du rapport azote nitrique/azote ammoniacal sur la croissance des talles et la coloration anthocyanique de cultivars de ray-grass; récemment, STREET et al. (1981) ont utilisé cette technique pour mettre en évidence l'effet de la silice sur la croissance de 3 espèces de graminées à gazon.

Afin de préciser les exigences en éléments minéraux majeurs des espèces de graminées à gazon les plus employées en France, une étude comparative a été conduite à l'aide de courbes de réponse de ces espèces aux 3 éléments (azote, phosphore, potassium), dans les conditions suivantes : cultures en solutions nutritives sur substrat inerte pendant les 4 premiers mois de croissance après le semis.

Les exigences en éléments minéraux d'un végétal peuvent être mises en évidence au moyen de courbes de réponse à l'azote, au phosphore, au potassium. Une courbe de réponse ou courbe d'action d'un élément nutritif est le graphe correspondant à la croissance du végétal en fonction des différentes concentrations de cet élément dans le milieu de culture, les autres éléments minéraux étant apportés en quantité suffisante (HELlER, 1969). Une étude antérieure (LEMAIRE, 1975) avait permis, à l'aide de ces courbes, de comparer l'action de l'azote sur les parties aériennes et les racines des végétaux.

\section{MATÉRIEL ET MÉTHODES}

\section{A. Matériel végétal}

Six graminées à gazon ont été étudiées : Lolium perenne L. cv. "Manhattan », Festuca rubra L. cv. " Manoir », Poa pratensis L. cv. " Monopoly », Festuca arundinacea Schreb. cv. "Ludion", Phleum bertelonii L. cv. « Sport», Agrostis tenuis Sibth.

\section{B. Technique de culture}

\section{Description}

La culture est réalisée en solutions nutritives sur un substrat inerte. Le pot de culture d'un volume de un litre est relié par sa partie basale au moyen d'un tuyau souple à un flacon contenant la solution nutritive. Le substrat est constitué par du silex broyé (3-5 $\mathrm{mm}$ ) surmonté d'une couche de sable de Loire $(0,5-2 \mathrm{~mm})$ constituant le lit de semence. Silex et sable ont été préalablement lavés à l'acide chlorhydrique dilué, puis rincés à l'eau permutée jusqu'à disparition des ions chlorures.

L'aération indispensable du substrat est réalisée périodiquement à l'aide d'un dispositif appelé « châssis à bascule " (fig. 1), mû par un " moteur à eau " (fig. 2). La durée des phases d'aération et d'alimentations minérale et hydrique est réglable; dans les

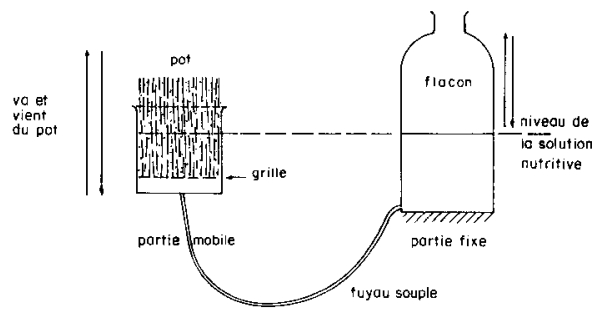

Figure 1

Principe du dispositif de culture en solutions nutritives sur substrat inerte.

Principle of experimental device for growing in liquid nutrient on an inert substrate.
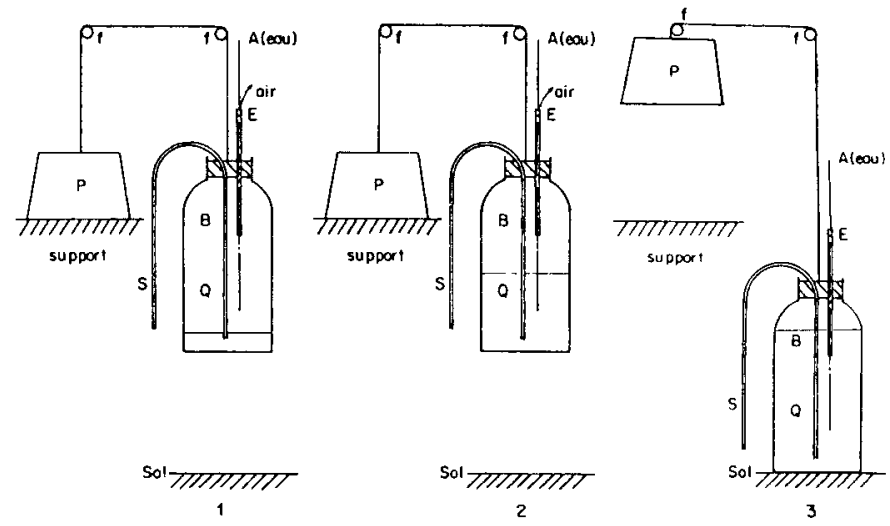

Figure 2

Description et fonctionnement du " moteur à eau ".

Description and working of the "water motor ».

$P$ : Poids de la nacelle et des pots

$f:$ Frottement des poulies

$B$ : Bonbonne

$S:$ Siphon

A : Tuyau d'arrivée d'eau

$E:$ Tube rigide recevant le tuyau $A$ de diamètre inférieur

$Q:$ Quantité d'eau dans la bonbonne

1. Nacelle et pots en position basse: $Q<P+f$. La bonbonne $B$ contient très peu d'eau.

2. Nacelle et pots en position basse: $Q<P+f$. La bonbonne $B$ se remplit d'eau; l'air s'en échappe grâce à la différence de diamètre entre $A$ et $E$.

3. Nacelle et pots en position haute $: Q>P+f$. La bonbonne $B$ tombe sur le sol; le siphon s'amorce par la compression de l'air restant obtenue par montée de l'eau dans la bonbonne et par suppression de l'échappement de l'air.

essais, le cycle de fonctionnement est de $10 \mathrm{mn}$ pour l'aération et de $40 \mathrm{mn}$ pour l'alimentation, soit 25 cycles/j.

\section{Solutions nutritives utilisées}

La composition de la solution nutritive (LEMAIRE, 1971) a été modifiée en fonction des concentrations en azote, phosphore ou potassium à réaliser (tabl. 1). Le rapport azote nitrique/azote ammoniacal est constant dans toutes les solutions nutritives pour empêcher une variation trop rapide du $\mathrm{pH}$ et une meilleure croissance des talles (NITTLER \& KENNY, 1976). Les traitements $\mathrm{N}, \mathrm{P}$ et $\mathrm{K}$ induisent respectivement des concentrations variables en $\mathrm{Ca}, \mathrm{Na}$ et $\mathrm{S}$ qui n'ont pas été compensées.

Le $\mathrm{pH}$ était compris entre 5,5 et 6,0 ; les conductivités variaient de $2,11 \mathrm{~ms}$ à $0,63 \mathrm{~ms}$. 
TABLEAU

Composition des solutions nutritives. Nutrient solution composition.

\begin{tabular}{|c|c|c|}
\hline Elément & Concentration en $\mathrm{mg} / 1$ & Forme d'apport \\
\hline $\mathrm{N}$ & $\begin{array}{l}12,5 \text { à } 300 \text { quand variable } N \\
150 \text { quand variables } P \text { ou } K\end{array}$ & $\begin{array}{l}2 / 3 \text { sous forme } \mathrm{N} \mathrm{NO}_{3}^{-} \text {de } \mathrm{Ca}\left(\mathrm{NO}_{3}\right)_{2} \text { et } \mathrm{NH}_{4} \mathrm{NO}_{3} \\
1 / 3 \text { sous forme } \mathrm{N} \mathrm{NH}_{4}^{+} \text {de } \mathrm{NH}_{4} \mathrm{NO}_{3}\end{array}$ \\
\hline $\mathbf{P}$ & $\begin{array}{l}1,0 \text { à } 17,2 \text { quand variable } P \\
8,6 \text { quand variables } N \text { ou } K\end{array}$ & $\mathrm{Na} \mathrm{H}_{2} \mathrm{PO}_{4}$ \\
\hline K & $\begin{array}{l}7,7 \text { à } 248 \text { quand variable } K \\
124 \text { quand variables } \mathrm{N} \text { ou } \mathrm{P}\end{array}$ & $\mathrm{K}_{2} \mathrm{SO}_{4}$ \\
\hline $\mathrm{Ca}$ & $\begin{array}{l}5,3 \text { à } 143 \text { quand variable } N \\
71,5 \text { quand variables } P \text { ou } K\end{array}$ & $\mathrm{Ca}\left(\mathrm{NO}_{3}\right)_{2}$ \\
\hline $\mathrm{Mg}$ & 6 & $\mathrm{Mg} \mathrm{SO}_{4}, 7 \mathrm{H}_{2} \mathrm{O}$ \\
\hline $\mathrm{Na}$ & $\begin{array}{l}0,8 \text { à } 12,8 \text { quand variable } P \\
6,4 \text { quand variables } \mathrm{N} \text { ou } \mathrm{K}\end{array}$ & $\mathrm{Na} \mathrm{H} \mathrm{PO}_{4}$ \\
\hline$S$ & $\begin{array}{l}11,1 \text { à } 110 \text { quand variable } K \\
59 \text { quand variables } N \text { ou } P\end{array}$ & $\mathrm{~K}_{2} \mathrm{SO}_{4}$ et $\mathrm{Mg} \mathrm{SO}_{4}, 7 \mathrm{H}_{2} \mathrm{O}$ \\
\hline Mo & 0,027 & \\
\hline B & 0,845 & \\
\hline $\mathrm{Mn}$ & 0,50 & sous forme de solution commerciale \\
\hline $\mathrm{Cu}$ & 0,0625 & comprenant tous ces oligo-éléments \\
\hline $\mathrm{Zn}$ & 0,227 & \\
\hline $\mathrm{Fe}$ & 0,60 & \\
\hline
\end{tabular}

\section{Essais réalisés}

\section{Disposition des traitements}

Les essais réalisés pendant 3 ans comportaient les 3 traitements : azote, phosphore, potassium sur les espèces expérimentées. Cinq doses de chacun des traitements sont appliquées avec 2 répétitions.

Chaque "châssis à bascule » reçoit une espèce et un traitement, de manière à obtenir des conditions identiques d'alimentation et d'aération. Des pots ont été placés aux extrémités afin de limiter l'effet de bordure. La répartition des 5 doses d'un traitement a été tirée au hasard pour un châssis.

\section{Conduite des essais}

- 2 densités de semis ont été utilisées suivant la grosseur des graines :

500 graines/pot pour Lolium, Festuca rubra et arundinacea,

1000 graines/pot pour Poa, Agrostis, Phleum.

- les essais ont été réalisés sous abri vitré équipé d'ouvrants latéraux et de faîtage entre le $1^{\text {er }}$ mai et le 15 août de chaque année.

- les tontes commencent 4 semaines après le semis et sont effectuées périodiquement tous les $10 \mathrm{j}$ pour Festuca rubra, tous les $7 \mathrm{j}$ pour les 5 autres espèces. La hauteur de coupe était de $3 \mathrm{~cm}$.

Après chaque tonte, les solutions nutritives sont changées pour éviter des variations du $\mathrm{pH}$ et de leur composition.

\section{Mesures effectuées}

Les poids de matière sèche produite par pot sont déterminés après séchage à l'étude à $60^{\circ} \mathrm{C}$ jusqu'à poids constant.

\section{Interprétation}

Pour chaque espèce de graminée, des courbes de réponse à l'azote, phosphore et potassium ont été construites à partir des données expérimentales (fig. 3 , 4,5 ).

Les besoins en un élément minéral d'une espèce donnée sont représentés par la concentration responsable du rendement optimum, ce qui correspond au maximum de fonctionnement physiologique de la plante.

La détermination de ces concentrations peut être réalisée empiriquement par voie graphique. Cette méthode, outre son imprécision, ne permet pas de porter un jugement sur les lois qui régissent les réponses des plantes aux facteurs de l'alimentation minérale. Il semble plus enrichissant d'ajuster les données expérimentales aux modèles employés pour l'étude de ces phénomènes : un modèle d'ajustement non linéaire a été préféré à un modèle polynominal car, à qualité équivalente d'ajustement, il fera intervenir un nombre plus faible de paramètres (TOMASSONE et al., 1983).

- Modèle $n^{\circ} 1$ : $Y=\left(1-B^{-x}\right)$. Dans ce cas, l'optimum de rendement $A$ n'est atteint qu'asymptotiquement et on s'intéressera ensuité à la réalisation de 

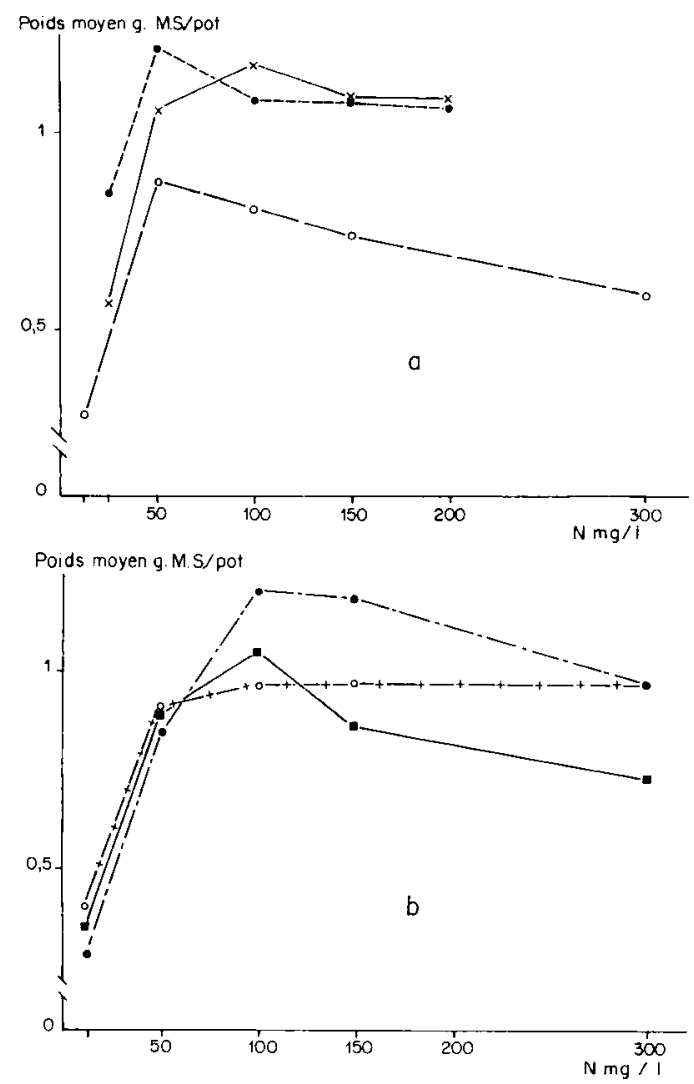

Figure 3

Courbes de réponse à l'azote.

Response curves to nitrogen.

a) Lolium perenne «Manhattan », Festuca rubra « Manoir ", Poa pratensis « Monopoly ».

b) Festuca arundinacea «Ludion », Phleum bertelonii "Sport », Agrostis tenuis.

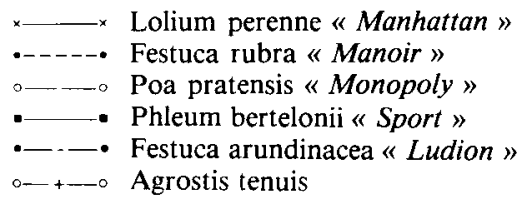

98 p. 100 de cet optimum. Le coefficient B est souvent appelé facteur d'efficacité.

- Modèle $n^{\circ} 2: Y=A\left(1-B^{-x}\right) C^{-x}$. Ce modèle exprime la prise en compte d'un effet toxique si le coefficient $\mathrm{C}$ est supérieur à 1 ; dans le cas où il est inférieur à 1 cela indique une stimulation qui est biologiquement peu vraisemblable dans ce contexte. D'autre part, l'optimum, fonction des B et C, peut être atteint expérimentalement.

Un calcul d'ajustement par les moindres carrés non linéaires a été effectué à l'aide du programme UWLONG (BACHACOU et al., 1981) en utilisant successivement les 2 modèles.

Au lieu d'effectuer un ajustement non linéaire sur les données expérimentales correspondantes aux 5 doses répétées 20 fois, le calcul a été réalisé, pour chaque coupe, sur la moyenne des valeurs de rendement obtenues dans les 2 répétitions de chacune des concentrations, pour les raisons suivantes :

- les résultats d'une coupe $n$ peuvent ne pas être indépendants de ceux de la coupe précédente n-1 ;
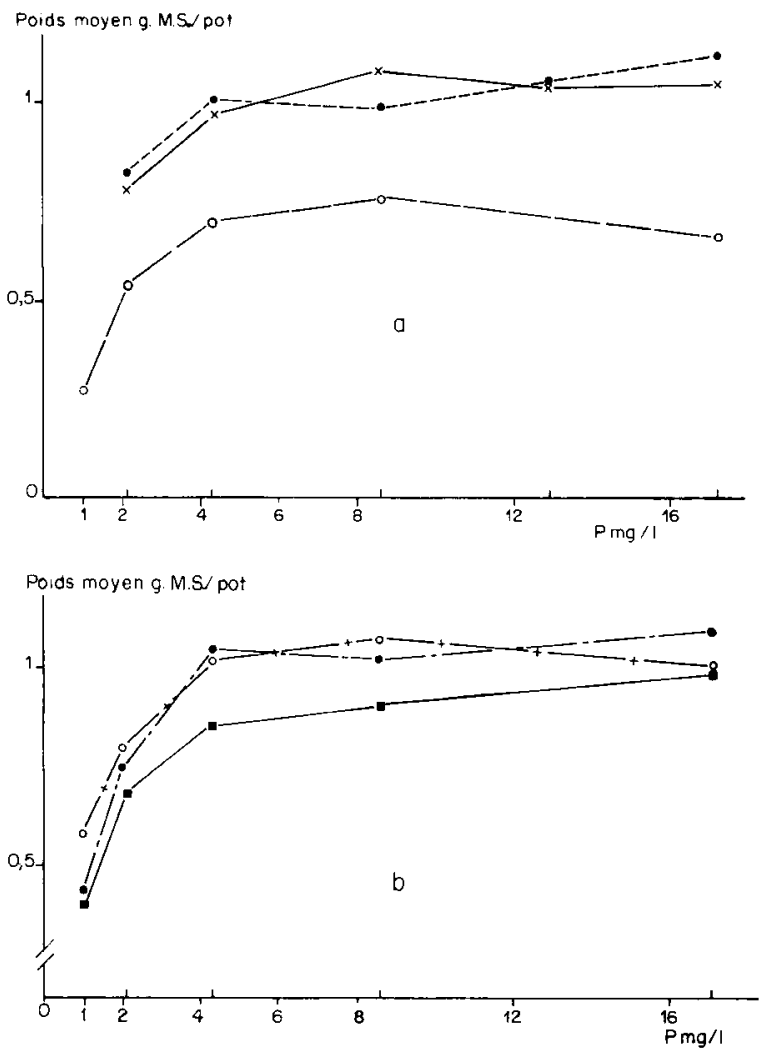

Figure 4

Courbes de réponse au phosphore (même légende que fig. 3).

Response curves to phosphorus (same legend as fig. 3).

a) Lolium perenne « Manhattan », Festuca rubra « Manoir », Poa pratensis « Monopoly ».

b) Festuca arundinacea "Ludion », Phleum bertelonii "Sport", Agrostis tenuis.
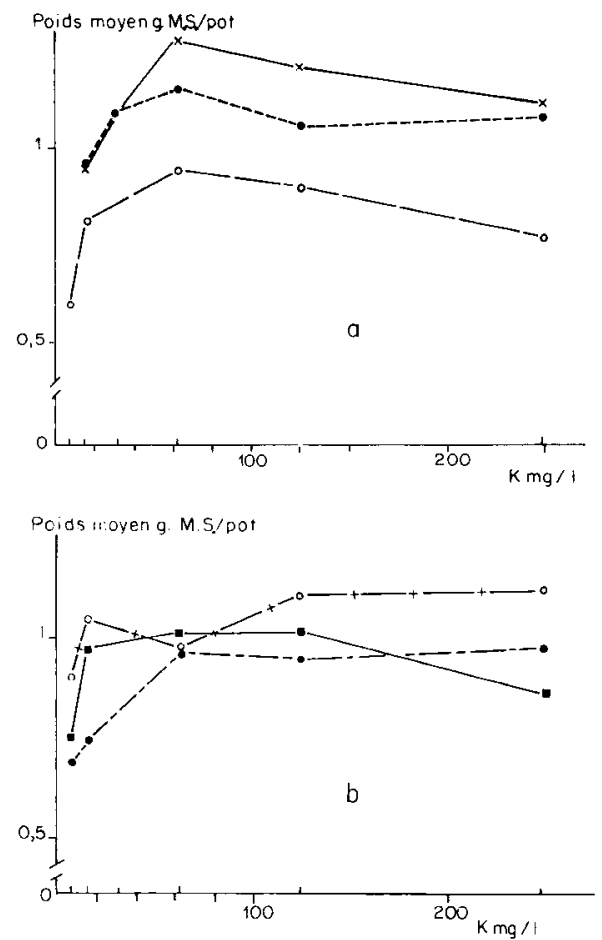

Figure 5

Courbes de réponse au potassium (même légende que fig. 3).

Response curves to potassium (same legend as fig. 3).

a) Lolium perenne "Manhattan », Festuca rubra " Manoir ", Poa pratensis « Monopoly".

b) Festuca arundinacea «Ludion », Phleum bertelonii « Sport », Agrostis tenuis. 
- un vieillissement du végétal peut intervenir dans les conditions de l'expérimentation bien que les observations aient été effectuées pendant les 4 premiers mois après le semis ;

- enfin les paramètres climatiques, bien que non limitants pendant la période de mai à août, peuvent exercer une action (LEMAIRE, 1975).
Pour chaque ajustement effectué, le coefficient de détermination $\mathrm{r}^{2}$ a été calculé.

Pour tester la validité du modèle, une estimation de la variance de l'erreur pure $\sigma^{2}$ a été calculée puisqu'on dispose de 2 répétitions par dose, 5 doses et 3 paramètres dans le modèle 2 - et a été comparée à la somme des carrés résiduelle après ajustement de la façon suivante :

$$
\begin{gathered}
\sum_{i=1}^{5} \sum_{j=1}^{2}\left(y_{i j}-f\left(x_{i}, \theta\right)\right)^{2}=\underbrace{2 \sum_{i=1}^{5}\left(y_{i o}-f\left(x_{i}, \theta\right)\right)^{2}}_{S_{1}}+\underbrace{\sum_{i=1}^{5} \sum_{j=1}^{2}\left(y_{i j}-y_{i o}\right)^{2}}_{S_{2}} \\
\sigma^{2} \chi_{10-3}^{2}=\sigma^{2} \chi_{5-3}^{2}+\sigma^{2} \chi_{10-5}^{2}
\end{gathered}
$$

d'où $\quad \hat{\sigma}^{2}=\frac{1}{5} \mathrm{~S}_{2}, \quad \grave{\text { a }} 5 \mathrm{ddl}$.

L'hypothèse "modèle exact 》 est conservée au niveau 5 p. $100 \mathrm{si}$

$\frac{S_{1 / 2}}{S_{2 / 5}}=\frac{\sum_{i=1}^{5}\left(y_{i o}-f\left(x_{i}, \theta\right)\right)^{2}}{\hat{\sigma}^{2}}$ est inférieure à $F_{2,5}$ au niveau 5 p. 100.

Après avoir éliminé les cas où l'hypothèse « modèle exact "n'a pas pu être conservée au niveau 5 p. 100 , l'ajustement avec le modèle 2 a été pris systématiquement quand le coefficient $\mathrm{C}$ était supérieur à 1 ; dans le cas contraire, le modèle simplifié a été utilisé.

Les concentrations responsables de l'optimum de rendement pour chacune des coupes ont été calculées de la façon suivante :

pour le modèle 2 ,

$$
\hat{\mathrm{X}}=\frac{\log \left(\frac{\log \hat{B}}{\log \hat{C}}+1\right)}{\log \hat{B}}
$$

et pour le modèle $1, \hat{\mathrm{x}}=-\frac{\log 0,02}{\log \hat{B}}$, en prenant comme dose optimum celle assurant 98 p. $100 \mathrm{du}$ rendement maximum A.

A partir de ces concentrations calculées pour chacune des 10 coupes, une concentration moyenne avec intervalle de confiance au niveau 5 p. 100 a été obtenue pour chaque élément minéral considéré et pour chaque espèce de graminée à gazon étudiée.

\section{RÉSULTATS}

\section{A. Courbes de réponse à l'azote}

Le coefficient de toxicité moyen $C$ est toujours supérieur à 1 quelle que soit l'espèce (tabl. 2). Les concentrations optimales ont donc été calculées d'après le modèle avec toxicité pour chacune des coupes (tabl. 3). Les concentrations moyennes permettent

TABLEAU 2

Valeurs moyennes des coefficients A-LnB-C calculés d'après les modèles 1 et 2 pour les 3 éléments minéraux et pour les 6 espèces de graminées pendant 10 coupes.

\begin{tabular}{|c|c|c|c|c|c|c|c|c|c|c|c|c|c|c|c|}
\hline \multirow{3}{*}{$\begin{array}{c}\text { Elément minéral } \\
\text { Modèle } \\
\text { Coefficient } \\
\end{array}$} & \multicolumn{5}{|c|}{$\mathbf{N}$} & \multicolumn{5}{|c|}{$\mathbf{P}$} & \multicolumn{5}{|c|}{$\mathrm{K}$} \\
\hline & \multicolumn{2}{|c|}{1} & \multicolumn{3}{|c|}{2} & \multicolumn{2}{|c|}{1} & \multicolumn{3}{|c|}{2} & \multicolumn{2}{|c|}{1} & \multicolumn{3}{|c|}{2} \\
\hline & A & LnB & A & $\operatorname{Ln} B$ & $\mathrm{C}$ & A & $\operatorname{Ln} B$ & A & $\operatorname{LnB}$ & $\mathrm{C}$ & $\mathbf{A}$ & $\operatorname{LnB}$ & A & LnB & $\mathrm{C}$ \\
\hline $\begin{array}{l}\text { Lolium perenne } \\
\text { «Manhattan » }\end{array}$ & 1,14 & 3,68 & 1,43 & 2,77 & 1,07 & 1,07 & 6,48 & 1,09 & 6,49 & 1,01 & 1,32 & 10,00 & 1,30 & 8,57 & 1,02 \\
\hline $\begin{array}{l}\text { Festuca rubra } \\
\text { « Manoir » }\end{array}$ & 1,27 & 6,75 & 1,36 & 5,38 & 1,05 & 1,26 & 6,88 & 1,03 & 10,03 & 0,96 & 1,14 & 11,94 & 1,06 & 12,37 & 1,00 \\
\hline $\begin{array}{l}\text { Poa pratensis } \\
\text { « Monopoly » }\end{array}$ & 0,76 & 5,41 & 0,90 & 4,47 & 1,05 & 0,74 & 5,88 & 0,84 & 4,58 & 1,08 & 0,85 & 13,90 & 0,95 & 14,12 & 1,03 \\
\hline $\begin{array}{l}\text { Phleum bertelonii } \\
\text { «Sport» }\end{array}$ & 0,84 & 5,04 & 1,08 & 3,83 & 1,04 & 1,02 & 5,47 & 0,99 & 5,70 & 0,99 & 1,23 & 15,93 & 1,10 & 17,28 & 1,04 \\
\hline $\begin{array}{l}\text { Festuca arundinacea } \\
\text { " Ludion }\end{array}$ & 1,18 & 2,91 & 1,52 & 1,83 & 1,06 & 1,15 & 5,61 & 1,10 & 5,72 & 1,02 & 1,08 & 11,86 & 1,05 & 17,51 & 0,99 \\
\hline Agrotis tenuis & 1,08 & 5,24 & 1,02 & 5,82 & 1,03 & 1,15 & 7,18 & 1,16 & 8,22 & 1,04 & 0,82 & 17,27 & 1,12 & 25,20 & 0,98 \\
\hline
\end{tabular}

Mean values of $A-\operatorname{Ln} B-C$ coefficients calculated with models 1 and 2 for the 3 mineral elements and for the 6 turfgrass species during 10 cuttings. 
TABLEAU 3

Doses optimales d' $N$ en $\mathrm{mg} / \mathrm{l}$ calculées pour les 6 espèces de graminées à gazon. Calculated optimum $N$ doses $(m g / l)$ for the 6 turfgrass species.

\begin{tabular}{|c|c|c|c|c|c|c|}
\hline $\mathrm{N}^{\circ}$ de la coupe & $\begin{array}{c}\text { Lolium } \\
\text { perenne } \\
\text { «Manhattan» }\end{array}$ & $\begin{array}{c}\text { Festuca } \\
\text { rubra } \\
\text { « Manoir» }\end{array}$ & $\begin{array}{c}\text { Poa } \\
\text { pratensis } \\
\text { «Monopoly » }\end{array}$ & $\begin{array}{c}\text { Phleum } \\
\text { bertelonii } \\
\text { «Sport » }\end{array}$ & $\begin{array}{c}\text { Festuca } \\
\text { arundinacea } \\
\text { « Ludion » }\end{array}$ & $\begin{array}{c}\text { Agrostis } \\
\text { tenuis }\end{array}$ \\
\hline 1 & $122(2)$ & 77 (2) & $105(2)$ & $105(2)$ & $194(2)$ & $186(1)$ \\
\hline 2 & $102(2)$ & $80(2)$ & $80(2)$ & $97(2)$ & $161(2)$ & $139(2)$ \\
\hline 3 & $111(2)$ & $94(2)$ & $88(2)$ & $105(2)$ & $145(2)$ & $103(2)$ \\
\hline 4 & $103(2)$ & $88(2)$ & $97(2)$ & $125(2)$ & $127(2)$ & $88(2)$ \\
\hline 5 & $133(2)$ & NS & $97(2)$ & 94 (2) & $114(2)$ & $86(2)$ \\
\hline 6 & $111(1)$ & 81 (2) & NS & $114(2)$ & $147(2)$ & 64 (1) \\
\hline 7 & $108(2)$ & $81(2)$ & $105(2)$ & $108(2)$ & $139(2)$ & $103(1)$ \\
\hline 8 & $116(2)$ & $69(2)$ & $88(2)$ & $103(2)$ & $153(2)$ & $47(1)$ \\
\hline 9 & $110(2)$ & N.C. & 77 (2) & $100(2)$ & $167(2)$ & $36(1)$ \\
\hline 10 & $101(2)$ & N.C. & $69(2)$ & $88(2)$ & $139(2)$ & $114(2)$ \\
\hline Moyenne & $122 \pm 2,5$ & $81,4 \pm 3,2$ & $89,5 \pm 3,6$ & $104 \pm 2,6$ & $149 \pm 5,6$ & $96,6 \pm 11,0$ \\
\hline
\end{tabular}

NS : valeur non retenue.

(1) : valeur obtenue par modèle 1 .

(2) : valeur obtenue par modèle 2 .

de comparer les différentes espèces de graminées entre elles et de les classer en fonction des doses optimum décroissantes: $F$. arundinacea $>L$. perenne $>$ $P$. bertelonii $>A$. tenuis $>P$. pratensis $>F$. rubra.

\section{B. Courbes de réponse au phosphore}

Le coefficient de toxicité moyen $\mathrm{C}$ atteint des valeurs supérieures à 1 seulement dans le cas de $P$. pratensis et d'A. tenuis (tabl. 2). Les concentrations optimales ont été calculées avec le modèle toxicité pour ces 2 espèces et avec le modèle simplifié pour les autres (tabl. 4). D'après les concentrations moyennes calculées, on peut classer les différentes espèces suivant l'ordre décroissant des besoins : $P$. pratensis $>F$. arundinacea, $P$. Bertelonii, L. perenne > A. tenuis, F. rubra.

\section{Courbes de réponse au potassium}

Le coefficient moyen de toxicité $C$ apparaît pour $P$. pratensis, $P$. bertelonii et $L$. perenne (tabl. 2 ). Le tableau 5 montre les concentrations calculées d'après l'un ou l'autre modèle suivant l'espèce considérée. Les concentrations moyennes permettent de classer les 6 espèces de façon décroissante $: L$. perenne $>P$. pratensis, $F$. rubra, $P$. bertelonii $>F$. arundinacea $>$ A. tenuis.

\section{DISCUSSION}

La détermination des concentrations minérales responsables des optimums de rendement permet de comparer les exigences des diverses espèces de grami-

TABLEAU 4

Doses optimales de $P$ en $\mathrm{mg} / \mathrm{l}$ calculées pour les 6 espèces de graminées à gazon.

Calculated optimum $P$ doses ( $\mathrm{mg} / \mathrm{l}$ ) for the 6 turfgrass species.

\begin{tabular}{|c|c|c|c|c|c|c|}
\hline $\mathrm{N}^{\circ}$ de la coupe & $\begin{array}{c}\text { Lolium } \\
\text { perenne } \\
\text { «Manhattan» }\end{array}$ & $\begin{array}{c}\text { Festuca } \\
\text { rubra } \\
\text { « Manoir » }\end{array}$ & $\begin{array}{c}\text { Poa } \\
\text { pratensis } \\
\text { «Monopoly » }\end{array}$ & $\begin{array}{l}\text { Phleum } \\
\text { bertelonii } \\
\text { «Sport» }\end{array}$ & $\begin{array}{c}\text { Festuca } \\
\text { arundinacea } \\
\text { « Ludion » }\end{array}$ & $\begin{array}{c}\text { Agrostis } \\
\text { tenuis }\end{array}$ \\
\hline 1 & $6,1(1)$ & 4,2 (1) & $9,5(2)$ & 11,7 (1) & 7,6 (1) & $2,5(1)$ \\
\hline 2 & $9,5(2)$ & $5,8(1)$ & $7,8(2)$ & 7,9 (1) & 6,8 (1) & 7,9 (1) \\
\hline 3 & 6,7 (1) & 6,4 (1) & $9,2(2)$ & 8,7 (1) & 6,4 (1) & 6,2 (1) \\
\hline 4 & 4,3 (1) & 7,4 (1) & $11,5(2)$ & 11,1 (1) & 7,8 (1) & 7,7 (2) \\
\hline 5 & 6,2 (1) & 4,7 (1) & $10,3(2)$ & 7,4 (1) & 4,3 (1) & $6,2(2)$ \\
\hline 6 & 6,9 (1) & 6,7 (1) & $8,4(2)$ & 5,9 (1) & 6,0 (1) & $5,6(2)$ \\
\hline 7 & $7,8(2)$ & 2,9 (1) & $7,2(2)$ & 6,4 (1) & 6,9 (1) & $6,8(2)$ \\
\hline 8 & $7,7(2)$ & 4,0 (1) & $7,9(2)$ & 5,1 (1) & 11,1 (1) & $4,1(2)$ \\
\hline 9 & $8,4(2)$ & N.C. & 7,1 (2) & 5,3 (1) & 10,7 (1) & 3,0 (2) \\
\hline 10 & $7,0(2)$ & N.C. & $6,7(2)$ & 2,5 (1) & 7,2 (1) & $6,8(2)$ \\
\hline Moyenne & $7,0 \pm 0,35$ & $5,2 \pm 0,52$ & $8,6 \pm 0,4$ & $7,4 \pm 0,68$ & $7,5 \pm 0,5$ & $5,7 \pm 0,47$ \\
\hline
\end{tabular}

NS : valeur non retenue.

(1) : valeur obtenue par modèle 1.

(2) : valeur obtenue par modèle 2. 
TABLEAU 5

Doses optimales de $K$ en $\mathrm{mg} / \mathrm{l}$ calculées pour les 6 espèces de graminées à gazon. Calculated optimum $K$ doses $(\mathrm{mg} / \mathrm{l})$ for the 6 turfgrass species.

\begin{tabular}{|c|c|c|c|c|c|c|}
\hline$N^{\circ}$ de la coupe & $\begin{array}{c}\text { Lolium } \\
\text { perenne } \\
\text { «Manhattan» }\end{array}$ & $\begin{array}{c}\text { Festuca } \\
\text { rubra } \\
\text { « Manoir » }\end{array}$ & $\begin{array}{c}\text { Poa } \\
\text { pratensis } \\
\text { "Monopoly » }\end{array}$ & $\begin{array}{c}\text { Phleum } \\
\text { bertelonii } \\
\text { «Sport » }\end{array}$ & $\begin{array}{c}\text { Festuca } \\
\text { arundinacea } \\
\text { "Ludion " }\end{array}$ & $\begin{array}{c}\text { Agrostis } \\
\text { tenuis }\end{array}$ \\
\hline 1 & $62(2)$ & NS & 48 (2) & 55 (2) & NS & $11(1)$ \\
\hline 2 & $68(2)$ & $17(1)$ & $36(2)$ & 48 (2) & $32(1)$ & $16(1)$ \\
\hline 3 & 71 (2) & $28(1)$ & $29(2)$ & $36(2)$ & 33 (1) & 17 (1) \\
\hline 4 & $68(2)$ & 38 (1) & $36(2)$ & 41 (2) & $13(1)$ & 19 (1) \\
\hline 5 & 74 (2) & $19(1)$ & $43(2)$ & $29(2)$ & $10(1)$ & $18(1)$ \\
\hline 6 & 57 (2) & $68(2)$ & $50(2)$ & 31 (2) & $16(1)$ & $16(1)$ \\
\hline 7 & $65(2)$ & $57(2)$ & $55(2)$ & $34(2)$ & $20(1)$ & 19 (1) \\
\hline 8 & $65(2)$ & $77(2)$ & $52(2)$ & $36(2)$ & 32 (1) & $36(1)$ \\
\hline 9 & $80(2)$ & N.C. & $55(2)$ & $38(2)$ & $29(1)$ & $20(1)$ \\
\hline 10 & $68(2)$ & N.C. & $59(2)$ & 27 (2) & 39 (1) & $10(1)$ \\
\hline Moyenne & $67,8 \pm 1,5$ & $43,4 \pm 9,8$ & $46 \pm 2,5$ & $37,5 \pm 2,1$ & $24,8 \pm 2,9$ & $18,2 \pm 1,7$ \\
\hline
\end{tabular}

NS : valeur non retenue.

(1) : valeur obtenue par modèle 1 .

(2) : valeur obtenue par modèle 2 .

N.C. : non calculé.

nées à gazon. Il apparaît que les classements des 6 espèces étudiées ne sont pas les mêmes suivant l'élément minéral considéré. Il ne faut pas considérer les chiffres en valeur absolue car ils ont peu de signification agronomique dans le domaine pratique; ils ne permettent pas de déterminer avec précision les quantités d'engrais à apporter pour effectuer une fertilisation correcte des gazons in situ. Ces résultats ont, par contre, le mérite de montrer que les différentes espèces de graminées à gazon ont des besoins différents, ce qui est d'autant plus intéressant que les gazons cultivés sont des mélanges de ces diverses espèces.

On peut remarquer que $P$. pratensis n'occupe pas la position attendue quant à ses exigences en azote.

Il faut aussi prendre en compte que ces résultats concernent l'installation de jeunes plantes pendant 4 mois après le semis ; il n'est pas certain que le classement des espèces soit le même avec des gazons installés depuis plus longtemps. Une réponse partielle peut être donnée au sujet de l'azote : à l'aide d'une expérimentation au champ sur les gazons monospécifiques installés depuis 2 ans, il a été montré que L. perenne cv. "Manhattan " était plus exigeant en azote que $P$. pratensis cv. "Monopoly 》 et $F$. rubra cv. « Highlight » (LEMAIRE, 1982).

De plus, on peut s'interroger sur la notion de besoins en éléments minéraux des gazons. Cette notion peut se définir :

- d'abord comme la satisfaction de l'optimum physiologique d'un peuplement monospécifique,

- comme la réalisation d'un équilibre entre les différentes espèces d'un mélange afin d'éviter une dérive de la composition floristique,

- puis comme le maintien d'un équilibre parties aériennes/racines suivant l'utilisation du gazon dans un but sportif ou ornemental (LEMAIRE, 1983),

- enfin comme la satisfaction des besoins en esthétique de l'utilisateur; ainsi apparait la notion de besoins minimum en un élément minéral, l'azote par exemple, pour l'obtention du meilleur aspect esthétique général (LEMAIRE, 1982).

\section{CONCLUSIONS}

Les 6 espèces de graminées à gazon observées ont des exigences minérales variées et peuvent être classées en 3 groupes suivant l'élément minéral considéré :

\begin{tabular}{llll}
\hline & Exigences fortes & \multicolumn{1}{c}{$\begin{array}{c}\text { Exigences } \\
\text { moyennes }\end{array}$} & Exigences faibles \\
$\mathrm{N}$ & $\begin{array}{l}\text { Festuca arundi- } \\
\text { nacea }\end{array}$ & $\begin{array}{l}\text { Lolium perenne } \\
\text { Phleum bertelonii }\end{array}$ & $\begin{array}{l}\text { Agrostis tenuis } \\
\text { Poa pratensis } \\
\text { Festuca rubra }\end{array}$ \\
\hline & Poa pratensis & $\begin{array}{l}\text { Phleum bertelonii } \\
\text { Festuca arundi- } \\
\text { nacea } \\
\text { Lolium perenne }\end{array}$ & $\begin{array}{l}\text { Agrostis tenuis } \\
\text { Lesubra }\end{array}$ \\
& Lolium perenne & $\begin{array}{l}\text { Festuca rubra } \\
\text { Poa pratensis } \\
\text { Phleum bertelonii }\end{array}$ & $\begin{array}{l}\text { Festuca arundi- } \\
\text { nacea } \\
\text { Agrostis tenuis }\end{array}$ \\
\hline
\end{tabular}

Une conséquence importante apparâit pour le raisonnement de la fertilisation des gazons, qui sont des peuplements plurispécifiques de graminées : difficulté de fixer une fertilisation azotée, phosphatée ou potassique qui puisse convenir à toutes les espèces.

Une autre conclusion peut aussi être dégagée : la composition des mélanges à gazon doit être réalisée avec des espèces ayant des exigences minérales similaires pour diminuer les compétitions entre espèces et pour la réalisation de la fertilisation convenable.

Le raisonnement d'une fertilisation correcte des gazons devrait être fait à l'avenir en tenant compte des exigences propres des espèces composant le gazon, des différents buts d'utilisation, ornemental ou sportif, et enfin des désirs de l'utilisateur quant au niveau d'aspect esthétique recherché. 


\section{REMERCIEMENTS}

Nous tenons à remercier P. VINCOURT (I.N.R.A. Lusignan) pour l'aide précieuse apportée pour le calcul des ajustements non linéaires.

\section{RÉFERENCES BIBLIOGRAPHIQUES}

Adams W. A., 1977. Effects of nitrogen fertilization and cutting height on the shoot growth, nutrient removal and turfgrass composition of a initially perennial ryegrass dominant sportsturf. Proc. 3rd Intern. Turfgrass Res. Conf., Munich, West Germany, 343-350. Bachacou J., Masson J. P., Millier C., 1981. Amance, manuel de la programmathèque statistique, I.N.R.A., Versailles, 516 p.

Beard J. B., 1973. Turfgrass Science and Culture. Prentice Hall Englewoods Cliffs, $\mathrm{n}^{\circ} 5,658 \mathrm{p}$.

Cairol D., Chevallier C., Bourgoin B., Monnet Y., 1982. Les surfaces engazonnées Bull. Tech. Inf. Min. Agric., 366, 39-55.

Christians N. E., Martin D. P., Karnok K. J., 1981. The interactions among $\mathrm{N}, \mathrm{P}, \mathrm{K}$, on the establishment, quality and growth of Kentucky bluegrass. Proc. 4th Intern. Turfgrass Res. Conf., Guelph, Canada, 341-348.

Dunn J. H., Nelson C. J., Winfrey R. D., 1981. Effects of mowing and fertilization on quality of ten Kentucky bluegrass cultivars. Proc. 4th Intern. Turfgrass Res. Conf., Guelph, Canada, 293-301.

Goss R. L., Law A. G., 1967. Performance of bluegrass varieties at two cutting heights and two nitrogen levels. Agron. J., 59, 516-518.

Heller R., 1969. Biologie végétale - Nutrition et métabolisme. Masson \& Cie, Ed. Paris, tome 1, 244 p.

Lee G. R., 1976. The response of seven perennial ryegrass varieties to nitrogen application. J. Br. Grassl. Soc., 13 (1), 42.

Lemaire F., 1971. Effet des fractions extraites de la matière organique sur la physiologie des plantes. Trans. intern. Symp. « Humus et Planta $V \geqslant-$ Prague, 511-518.

Lemaire F., 1975. Action comparée de l'alimentation azotée sur la croissance des racines et des parties aériennes des plantes. Ann. Agron., 26 (1), 59-74.
Lemaire F., 1982. Contribution à une meilleure connaissance de la relation fertilisation azotée-aspect esthétique des gazons. Agronomie, 2 (8), 765-772.

Lemaire F., 1983. Sur l'équilibre parties aériennes/racines. Cas des graminées à gazon. C. $R$. Séminaire d'études sur la Physiologie de la plante entière. Chambon-du-Lac. Publ. I.N.R.A., Département d'Agronomie. Sous presse.

Madison J. H., 1962. Turfgrass ecology. Effects of mowing, irrigation and nitrogen treatments on Agrostis tenuis Huds. "Seaside" and Agrostis tenuis Sibth. " Highland " on population, yield, rooting and cover. Agron. J., 54, 407-412.

Minderhoud J. W., 1976. Triebformen einiger Rasengräser und ihre Beeinflüssung durch Pflege und Benützung. Rasen, Turf, Gazon, 3, 80-84.

Nittler L. W., Kenny T. J., 1976. Effect of ammonium to nitrate ratio on growth and anthocyanin development of perennial ryegrass cultivars. Agron. J., 68 (4), 680-682.

Schweizer E. W., 1974. Erhebungen über den Nährstoffentzung verschiedener Rasengräser und Rasengrasmischungen im Verlaufe der Vegetationsperiode. Rasen, Turf, Gazon, 5 (3), 65-68.

Street J. R., Henderlong P. R., Himes F. L., 1981. The effect of silica rates on the growth, silica and water absorption among three turfgrass species. Proc. 4th Intern. Turfgrass Res. Conf., Guelph, Canada, 259-266.

Tomassone R., Lesquoy E., Millier C., 1983. La Régression. Nouveaux regards sur une ancienne méthode statistique. Actualités scientifiques et agronomiques I.N.R.A., Masson Ed., 180 p. 